\title{
A Self-Programmable Interface for WSN Applications
}

\author{
S.D. Vera, A. Bayo, N. Medrano, B. Calvo, S. Celma \\ Group of Electronic Design (GDE) \\ Aragón Institute of Engineering Research (I3A) \\ University of Zaragoza, Pedro Cerbuna 12, 50009, Zaragoza, Spain. \\ Tel.+34-976761240, Fax+34-976762143, e-mail: svera@unizar.es
}

\begin{abstract}
Numerous applications of Wireless Sensor Networks (WSN) involve monitoring physical and chemical parameters in large regions, thus needing a large number of sensor nodes. In order to reduce the cost of these nodes, it is usual to use low-cost analogue sensors followed by a programmable electronic interface capable of adapting every sensor output to the port requirements of the microcontroller embedded in the sensing node. The goal of the present work is the design and test of a low-voltage plug\&play programmable sensor-to-microcontroller interface able to self-configure its operation when adapting the output of different sensors, achieving an optimum reading performance for every sensor. The proposed interface, which includes both electronic and software elements, is shown in Figure 1a. By properly programming the interface electronics, the system can be used to conditioning active and passive sensors, enabling plug\&play to be easily integrated in a WSN node and taking advantage of the full span of the connected device. It provides a value of the measured parameter coded as the frequency of a signal compatible with the logic levels of the master microcontroller. Experimental test results to validate its performance are given for a resistive humidity sensor (Figure 1b) and other low-cost sensors. By properly managing the interface electronics, the average power consumption in a measurement process of the conditioning electronics remains low.
\end{abstract}

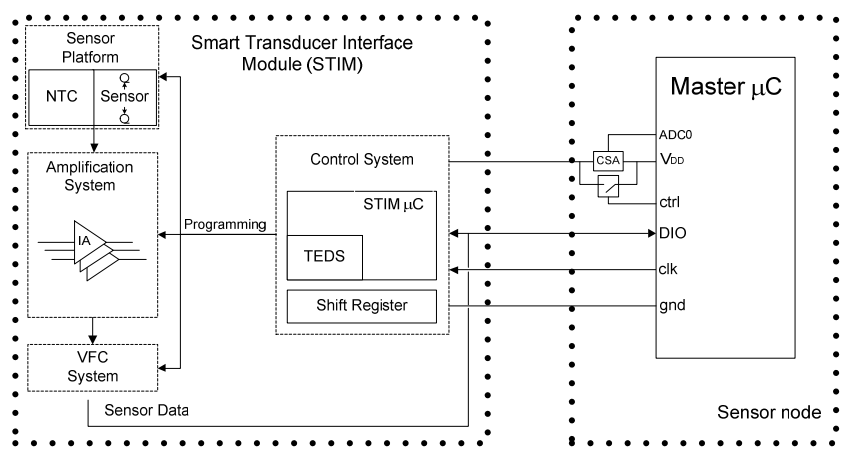

(a)

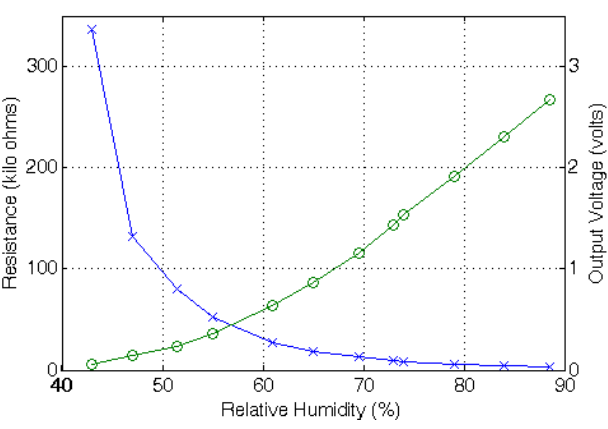

(b)

Figure 1: (a) Complete scheme diagram of proposed sensor interface and communications. (b) Humidity sensor behavior ( $\mathrm{x}$ ) compared to the output voltage read from the master microcontroller (o) at $26^{\circ} \mathrm{C}$. 